\title{
A note on utilising binary features as ligand descriptors
}

\author{
Hamse Y. Mussa ${ }^{1,2^{*}}$, John B. O. Mitchell ${ }^{2}$ and Robert C. Glen ${ }^{1}$
}

\begin{abstract}
It is common in cheminformatics to represent the properties of a ligand as a string of 1's and 0's, with the intention of elucidating, inter alia, the relationship between the chemical structure of a ligand and its bioactivity. In this commentary we note that, where relevant but non-redundant features are binary, they inevitably lead to a classifier capable of capturing only a linear relationship between structural features and activity. If, instead, we were to use relevant but non-redundant real-valued features, the resulting predictive model would be capable of describing a non-linear structure-activity relationship. Hence, we suggest that real-valued features, where available, are to be preferred in this scenario.
\end{abstract}

Keywords: Binary descriptors, Ligand chemical structure, Linear relationship, Bernoulli distribution

\section{Background}

One of the major goals of cheminformatics is to predict the relationship between a ligand's chemical structure and its bioactivity [1]. If this relationship is captured correctly, then (among other goals) designing the right drug for each disease would become an easier task $[1,2]$. Unfortunately, the structure-activity relationship can often be intricate and arcane, and in particular non-linear.

To devise an adequate model describing this relationship, the cheminformaticist typically follows a standard approach; starting with a large number of ligand attributes or features considered important for representing the underlying characteristics of the ligand, and relevant to its bioactivity. Then, through feature selection techniques, one selects the ligand attributes deemed to have statistically minimum interdependence among themselves (given the ligand bioactivity), while also showing strong association with the ligand bioactivity [3-5]. With this step, one strives for a set of relevant but nonredundant ligand features $[4,5]$ : "relevant" in the sense

\footnotetext{
*Correspondence: mussax021@gmail.com

${ }^{1}$ Centre for Molecular Informatics, Department of Chemistry,

Cambridge University, Lensfield Road, Cambridge CB2 1EW, UK

Full list of author information is available at the end of the article
}

that there is a strong association between the selected features and the bioactivity, and "non-redundant" in the sense that these features are conditionally independent given the bioactivity. (Irrelevant features are basically noise and relevant but redundant features are nuisance [6]; we are not concerned with these features here [6]).

Typically the ligand's chemical structure is represented by an $L$-dimensional vector $\mathbf{x}=\left(x_{1}, x_{2}, \ldots, x_{L}\right)$. The elements $x_{l}$ ideally contain appropriate information about the ligand's features, relevant for predicting its bioactivity. This bioactivity against a particular target or protein may be represented either numerically or as a class label; such classes (or class labels) are denoted henceforth by $k$, where $k=1,2, \ldots, K$ with $K$ being the total number of classes of interest.

Identifying the relevant features $\mathbf{x}$ without errors is generally impossible. Usually both $\mathbf{x}$ and $k$ are treated as random variables such that for a given $\mathbf{x}$ we have a distribution $p(k \mid \mathbf{x})$-the so-called class posterior probability-on the different possible classes [1, 7]. In practice, $p(k \mid \mathbf{x})$ that can assign a new ligand represented by $\mathbf{x}$ to the class minimising the probability of misclassification is induced from given prototype samples (a training dataset) $[8,9]$.

In Bayesian probabilistic settings, it is usually computationally easier to estimate $p(k \mid \mathbf{x})$ in terms of class

\section{Chemistry Central}

(C) 2015 Mussa et al. This article is distributed under the terms of the Creative Commons Attribution 4.0 International License (http://creativecommons.org/licenses/by/4.0/), which permits unrestricted use, distribution, and reproduction in any medium, provided you give appropriate credit to the original author(s) and the source, provide a link to the Creative Commons license, and indicate if changes were made. The Creative Commons Public Domain Dedication waiver (http://creativecommons.org/ publicdomain/zero/1.0/) applies to the data made available in this article, unless otherwise stated. 
probability $(p(k))$, evidence $(p(\mathbf{x}))$ and class-conditional probability density function $(p(\mathbf{x} \mid k))$ :

$$
p(k \mid \mathbf{x})=p(\mathbf{x} \mid k) \times \frac{p(k)}{p(\mathbf{x})},
$$

In cheminformatics, the main task of estimating $p(k \mid \mathbf{x})$ often reduces to inducing $p(\mathbf{x} \mid k)$ from the training dataset.

\section{Commentary}

It is common practice nowadays to assume that the $L$ relevant chemical structure features of the ligand can be encoded as a binary "vector" of 1's and 0's denoting presence (1) and absence ( 0 ) of these features-i.e., $x_{l} \in\{0,1\}$ [10]. In practice, state-of-the-art feature selection techniques $[3,5]$ that are based on information theory are used to quantify the level of association between the features and the bioactivity. These techniques are also capable of quantifying the class-conditional interdependency among the features. However, in the light of the insightful work of Li on the peculiar but useful characteristics of the conditional dependence between two binary random variables [11], one might be able to go one step further; identify the $L^{\prime}$ features in the $L$ relevant features whose relationship with the bioactivity is statistically significant, but whose class-conditional interdependency is statistically insignificant-i.e., retain features that are statistically non-redundant (and for that matter ignore or discard statistically redundant features).

In our probabilistic setting, $L^{\prime}$ relevant descriptors $\mathbf{x}^{\prime}=\left(x_{1}^{\prime}, x_{2}^{\prime}, \ldots, x_{L}^{\prime}\right)$ being non-redundant entails that $p\left(\mathbf{x}^{\prime} \mid k\right)$ can be expressed as a product of $L^{\prime}$ class-conditional univariate probability density functions $p\left(x_{l}^{\prime} \mid k\right)$, i.e., $p\left(\mathbf{x}^{\prime} \mid k\right)=\Pi_{l=1}^{L^{\prime}} p\left(x_{l}^{\prime} \mid k\right)$. This means that $p\left(k \mid \mathbf{x}^{\prime}\right)$, which is what we are interested in estimating, can be given as

$$
p\left(k \mid \mathbf{x}^{\prime}\right)=\Pi_{l=1}^{L^{\prime}} p\left(x_{l}^{\prime} \mid k\right) \times \frac{p(k)}{p\left(\mathbf{x}^{\prime}\right)},
$$

Since $x_{l}^{\prime} \in\{0,1\}$, the univariate distributions $p\left(x_{l}^{\prime} \mid k\right)$ are Bernoulli $[8,12,13]$, i.e. $p\left(x_{l}^{\prime} \mid k\right)=p\left(x_{l}^{\prime}=1 \mid k\right)^{x_{l}^{\prime}}$ $\left[1-p\left(x_{l}^{\prime}=1 \mid k\right)\right]^{\left(1-x_{l}\right)}$. In terms of these Bernoulli distributions, Eq. 2 modifies to

$$
\begin{aligned}
p\left(k \mid \mathbf{x}^{\prime}\right)= & \Pi_{l}^{L^{\prime}} p\left(x_{l}^{\prime}=1 \mid k\right)^{x_{l}^{\prime}}\left[1-p\left(x_{l}^{\prime}=1 \mid k\right)\right]^{\left(1-x_{l}\right)} \\
& \times \frac{p(k)}{p\left(\mathbf{x}^{\prime}\right)}
\end{aligned}
$$

which can be further rewritten in an equivalent but more convenient form (see Chapter 4 of ref [8]):

$$
g_{k}\left(\mathbf{x}^{\prime}\right)=\sum_{l}^{L^{\prime}} x_{l}^{\prime} c_{k l}+d_{k},
$$

where $\quad c_{k l}=\log \frac{p\left(x_{l}^{\prime} \mid k\right)}{1-p\left(x_{l}^{\prime} \mid k\right)} ; \quad d_{k}=\sum_{l=1}^{L^{\prime}} \log \left(1-p\left(x_{l}^{\prime} \mid k\right)\right)+$ $\log \frac{p(k)}{p\left(\mathbf{x}^{\prime}\right)}$. Clearly, the discriminant function $g_{k}\left(\mathbf{x}^{\prime}\right)$ is linear in $\mathbf{x}^{\prime}[8,12,13]$-irrespective of the nature of the association between the chemical structure of the ligand and its bioactivity. This is the consequence of the ligand's relevant but non-redundant features being represented by a binary "vector".

However, the situation can be different if non-redundant real-valued features are utilised to represent the chemical structure of the ligand. In this scenario the $L^{\prime}$ class-conditional univariate distributions $p\left(x_{l}^{\prime} \mid k\right)$ are not necessarily Bernoulli. Here $p\left(x_{l}^{\prime} \mid k\right)$ can be expressed in Hermite polynomial basis functions $\phi_{n}\left(x_{l}^{\prime}\right)$ in variable $x_{l}^{\prime}$

$$
p\left(x_{l}^{\prime} \mid k\right)=\sum_{n}^{\infty} \alpha_{n l}^{k} \phi_{n}^{k}\left(x_{l}^{\prime}\right),
$$

where $\alpha_{n l}^{k}$ are the appropriate coefficient values. Note that the $k$ in $\alpha_{n l}^{k}$ and $\phi_{n}^{k}$ is just an index (not a power). Inserting Eq. 5 into Eq. 2 and then taking the logarithm of the resultant equation yields the following discriminant function

$$
h_{k}\left(\mathbf{x}^{\prime}\right)=\sum_{l}^{L^{\prime}}\left[\sum_{n}^{\infty} \alpha_{n l}^{k} \phi_{n}^{k}\left(x_{l}^{\prime}\right)\right]+b_{k},
$$

where $b_{k}=\log \frac{p(k)}{p\left(\mathbf{x}^{\prime}\right)}$. Clearly $h_{k}\left(\mathbf{x}^{\prime}\right)$ is not necessarily linear in $\mathbf{x}^{\prime}$ even though the $L^{\prime}$ features utilised are classconditionally independent [13]. Thus, for real-valued features, the resulting classifier is capable of representing a non-linear structure-activity relationship.

\section{Conclusions}

In this commentary it has been noted that, when ligand features are represented by a string of binary numbers, one must end up with a linear model for describing the dependency (if any) between the chemical structure of a ligand and its bioactivity of interest-albeit in a classification setting. Such a linear model may be severely biased and limited in its predictivity. It was also pointed out that, where relevant real-valued features are used, the resulting model can be unbiased as it can adequately capture both linear and non-linear structure-activity relationships.

\section{Authors' contributions}

HYM (with the help of JBOM) initiated the theme-questioning the validity of representation of ligand chemical structure as a $L$ long fingerprint to study the relationship between the chemical structure of a ligand and its bioactivity. $\mathrm{RCG}$ checked the consistency of the theme in regard to drug development. All authors contributed to writing and revising the final draft of the manuscript.

All authors read and approved the final manuscript. 


\section{Author details}

${ }^{1}$ Centre for Molecular Informatics, Department of Chemistry, Cambridge University, Lensfield Road, Cambridge CB2 1EW, UK. ${ }^{2}$ EaStCHEM School of Chemistry and Biomedical Sciences Research Complex, University of St Andrews, North Haugh, St Andrews, KY16 9ST St Andrews, Scotland.

\section{Acknowledgements}

Mussa would like to thank the Centre for Molecular Informatics for using their facilities. Mussa and Mitchell thank the BBSRC for funding this research through grant BB/I00596X/1. Mitchell thanks the Scottish Universities Life Sciences Alliance (SULSA) for financial support.

\section{Competing interests}

The authors declare that they have no competing interests.

Received: 14 August 2015 Accepted: 11 November 2015

Published online: 01 December 2015

\section{References}

1. Mussa HY, Marcus D, Mitchell JBO, Glen RC (2015) Verifying the fully "Laplacianized" Naive Bayes and more. J Cheminform 7:27

2. Afzal AM, Mussa HY, Turner RE, Bender A, Glen RC (2015) A multi-label approach to target prediction taking ligand promiscuity into account. J Cheminform 7:24
3. Tourassia GD, Frederick ED, Markey MK, Floyd CE Jr (2001) Application of the mutual information criterion for feature selection in computer-aided diagnosis. Med Phys 28:2394-2402

4. Battiti R (1994) Using mutual information for selecting features in supervised neural net learning. IEEE Trans Neur Nets 5:537-550

5. Peng $\mathrm{H}$, Long F, Ding C (2005) Feature selection based on mutual information: criteria of max-dependency, max-relevance, and min-redundancy. IEEE Trans Patt Anal Mach Intel 27:1226-1238

6. Langley P, Sage S (1994) Induction of selective Bayesian classifiers. In: UAI94 Proceedings of the Tenth International Conference on Uncertainty in Artificial Intelligence, San Francisco, pp 399-406

7. Mussa HY, Afzal AM, Mitchell JBO (2015) Parzen Window approach reduced to two vectors and one matrix. Pat Recogn Lett 65:30

8. Duda RO (1973) Pattern classification and scene analysis, 1st edn. John Wiley \& Sons Ltd, New York

9. Young TY, Calvert TW (1974) Classification, estimation, and pattern recognition. Elsevier Science, New York

10. Willett $P$ (2011) Chemoinformatics: a history. Comput Mol Sci 1:46-56

11. Li W (1990) Mutual information function versus correlation functions. Stat Phys 60:823-837

12. Bahadur R R: A representation of the joint distribution of the responses to $n$ dichotomous items. In Studies in Item Analysis and Prediction; ed. Salomon H: Standford University Press: Standford, CA, 1961

13. Hand DJ, Yu K (2001) Idiot's Bayes-not ao stupid after all? Int Stat Rev 69:385-398

\section{Publish with ChemistryCentral and every scientist can read your work free of charge \\ "Open access provides opportunities to our colleagues in other parts of the globe, by allowing anyone to view the content free of charge."}

W. Jeffery Hurst, The Hershey Company.

- available free of charge to the entire scientific community

- peer reviewed and published immediately upon acceptance

- cited in PubMed and archived on PubMed Central

- yours - you keep the copyright

Submit your manuscript here:

htp://wWW.chemistrycentral.com/manuscript/ 